definition of 'fuckbuddy' in MSM population and to assess the risk factors for having a fuckbuddy.

Methods We conducted a mixed method study among MSM at the Melbourne Sexual Health Centre, Australia, between March and September 2015. (1) MSM attending MSHC during the study period were invited to complete a questionnaire about their regular and casual partnerships in the last three months. (2) Semi-structured interviews were conducted with 30 MSM who were asked their views on the terminology they used to describe their relationships and sexual partners.

Results A total of 939 MSM completed the questionnaire and 502 MSM had at least one regular partner, with a total of 1139 regular partnerships reported. The majority of regular partners were classified as 'fuckbuddies' (60\% [95\% CI 57\%63\%], 686/1139) s', followed by 'partners' (16\% [95\% CI $14 \%-18 \%])$ and 'boyfriends' (16\% [95\% CI $14 \%-18 \%])$. MSM who had at least one 'fuckbuddy' were 2.4 (95\% CI $1.29,4.41)$ times more likely to acquire rectal chlamydia after adjusting for total number of partners and condom use. Findings from interviews showed that the there was a consensus among men that partners they engaged with for 'sex only' were classified as casual partners, and partners with whom there was an emotional attachment or formalisation of the relationship, were classified as 'regular partners'. However, the classification of 'fuckbuddy' as a regular or casual partner was less clear.

Conclusion MSM with 'fuckbuddies' are at greater risk of acquiring STIs such as rectal chlamydia. Further research is needed to ascertain the ways in which men conceptualise sexual relationships and define or classify partner types, particularly 'fuckbuddy' relationships. A third category for sexual relationships should be considered to encapsulate fuckbuddy relationships.

\subsection{IT'S JUST NOT FOR ME: EXPLORING LOW PREP UPTAKE AMONG YOUNG BLACK MEN WHO HAVE SEX WITH MEN IN THE SOUTHERN UNITED STATES}

${ }^{1}$ Emily S Pingel, ${ }^{1}$ Charlotte-Paige Rolle, ${ }^{1}$ Colleen Kelley, ${ }^{1}$ Eli Rosenberg, ${ }^{2}$ Rob Stephenson, ${ }^{1}$ Patrick Sullivan, ${ }^{1}$ Aaron Siegler. ${ }^{1}$ Emory University, Atlanta, USA; ${ }^{2}$ University of Michigan, Ann Arbour, USA

\subsection{6/sextrans-2017-053264.9}

Introduction Pre-exposure prophylaxis (PrEP) is highly effective in preventing HIV acquisition. In the Southern United States, where young Black men who have sex with men (YBMSM) have the highest rates of new HIV infection, PrEP uptake remains low. As part of a longitudinal cohort study, YBMSM were offered optional, non-incentivized PrEP as a standard of HIV prevention care service. Among those who declined PrEP, we sought to understand their motivations, as well as their overall perceptions of PrEP as a prevention tool.

Methods The EleMENt study is an observational HIV/STI incidence cohort of HIV-negative YBMSM aged 18-29 years in Atlanta, Georgia. We conducted 24 in-depth, semi-structured interviews with men who declined optional PrEP offered by the study. Topics included PrEP knowledge, attitudes, and intentions. We employed a phenomenological lens to identify common themes in participant accounts of the decision to forgo PrEP.

Results Participants fell into two categories of PrEP refusal: those who indicated no current or future interest ("nevers") and those ambivalent about taking PrEP, but who had thus far not filled a prescription ("maybes"). YBMSM in both groups expressed mistrust of biomedical interventions, and despite being indicated for PrEP, often perceived themselves as low risk for HIV acquisition. They employed “othering” strategies, in which PrEP was described as appropriate for individuals in serodiscordant partnerships or with many casual partners. They viewed taking a daily pill as a burdensome measure only appropriate for extremely high-risk men (i.e., "the risky Other"). These perceptions were accentuated by instances of family members actively discouraged participants from taking PrEP.

Conclusion We discuss the role of future research exploring low risk estimation among YBMSM as a potential site of resistance to a public health designation of "high risk" amidst historical legacies of medical mistrust in Black communities. Such concerns must be addressed to design effective HIV and PrEP-specific interventions for this population.

\section{O02.5 NARRATING RISK: NEW AND TRADITIONAL METHODS TO UNDERSTAND SEXUAL RISK BEHAVIOUR AMONG HIV-UNINFECTED MEN WHO HAVE SEX WITH MEN IN LIMA, PERU}

${ }^{1}$ Jesse L Clark, ${ }^{2}$ Ximena Salazar, ${ }^{3}$ Williams Gonzales, ${ }^{4}$ Amaya Perez-Brumer, ${ }^{5}$ Francisco Nanclares, ${ }^{1}$ Eddy R Segura, ${ }^{5}$ James Dilley, ${ }^{3}$ Robinson Cabello. ${ }^{1}$ David Geffen School of Medicine At The University of California, Los Angeles, Los Angeles, USA; ${ }^{2}$ Universidad Peruana Cayetano Heredia, Lima - Peru; ${ }^{3}$ Asociacion Civil Via Libre, Lima Peru; ${ }^{4}$ Columbia University Mailman School of Public Health, New York, USA; ${ }^{5}$ University of California, San Francisco School of Medicine, San Francisco, USA

\subsection{6/sextrans-2017-053264.10}

Introduction Traditional risk-reduction counselling has had limited effect in modifying patterns of high-risk sexual behaviour among MSM. New methods like Personalised Cognitive Counselling (PCC) can be used to understand and address contexts of HIV transmission risk.

Methods We conducted interviews and focus groups with HIV-uninfected MSM in 3 stages: I) 4 FGs $(n=38)$ to explore community norms of male sexual interaction, HIV/STI testing practices, and acceptability of PCC; II) Interviews $(n=15)$ where MSM narrated and reflected on a recent experience of receptive condomless anal intercourse (CAI) with an HIVinfected or unknown status partner; and III) 3 FGs $(n=29)$ to discuss composite narratives of sexual risk constructed from Stage II interviews.

Results In exploratory FGs, fear was the guiding principle of HIV counselling/testing. CAI was commonly reported, HIV status rarely discussed, and testing decisions motivated by fear of recent infection. Counselling interactions were described as robotic, repeating stale information in encounters where patients were routinely stigmatised, criticised for engaging in CAI, and threatened with inevitable seroconversion. Negative results were considered to validate prior sexual practices, which then continued unchanged. Stage II interviews used narratives to articulate cognitive processes, partnership interactions, and social contexts where CAI was tacitly encouraged. Limited access to condoms, alcohol prior to sex, and preferences for "bare" sex were cited as justifications for CAI. When common narrative elements were re-presented to Stage III FGs as composite vignettes, participants reverted to standard counselling recommendations, mandating condom use and regular HIV/STI testing, without acknowledging disjunctions between the guidelines and their lived experiences. 
Conclusion In contrast to static information transfer, narrative techniques to reconstruct and reflect on recent encounters provide depth and relevance to counselling interactions, addressing multiple dimensions of HIV/STI risk experienced by MSM in Latin America.

\subsection{BEHAVIOURAL CHANGE TO REDUCE THE RISK OF PHARYNGEAL GONORRHOEA IN MEN WHO HAVE SEX WITH MEN}

Eric PF Chow, Sandra Walker, Tiffany Phillips, Christopher K Fairley. Melbourne Sexual Health Centre

\subsection{6/sextrans-2017-053264.11}

Introduction Gonorrhoea rate continues to increase at alarming rates among men who have sex with men (MSM), particularly in young MSM, in Australia and worldwide. A recent study has shown that use of antiseptic alcohol-containing mouthwash can inhibit the growth of gonorrhoea in the pharynx. The aim of this study was to examine the willingness of MSM to change their behaviours to reduce the risk of gonorrhoea.

Methods A cross-sectional questionnaire-based study was conducted among MSM attending the Melbourne Sexual Health Centre, Australia, between March and September 2015. Participants were asked how likely they would change their behaviours to reduce the risk of pharyngeal gonorrhoea. Six different strategies were asked: (1) stop tongue kissing with partners; (2) stop having receptive oral sex from partners (partner's penis in participant's mouth); (3) stop rimming partners (participant's tongue in or around partner's anus); (4) stop using saliva as lube for anal sex; (5) use condoms for oral sex; and (6) use alcohol-containing mouthwash daily.

Results A total of 926 MSM were surveyed with a median age of 29 (IQR 25-36) years. The majority of MSM $(65 \%$ [95\% CI 62\%-69\%]) expressed they were likely to use mouthwash daily to reduce the risk of pharyngeal gonorrhoea, followed by stop using saliva as lube $(63 \%$ [95\% CI $60 \%-$ 66\%]), and stop performing rimming (49\% [95\% CI 46\%$53 \%])$. In contrast, the majority of MSM (78\% [95\% CI $75 \%-80 \%])$ expressed they were unlikely to stop kissing to reduce the risk of pharyngeal gonorrhoea. Young MSM were more likely to use mouthwash daily to reduce the risk of gonorrhoea compared to older MSM.

Conclusion Men are less likely to change their sexual practices to reduce the risk of pharyngeal gonorrhoea however they are likely to use mouthwash daily to reduce the risk, particularly younger MSM.

\section{Oral Presentation Session 3}

\section{STI Prevention/Diagnosis and Community Engagement}

\subsection{AIDS PREVENTION POLICY AT SCHOOL IN BRAZIL (1994-2014) AND THE ROLE OF UNESCO}

${ }^{1}$ Luis Carlos Romero, 1,2Mariana Braga Neves, 'Maria Arebeca Otero Gomes. 'UNESCO; ${ }^{2}$ SBRASH

10.1136/sextrans-2017-053264.12
Introduction Information, values and knowledge transmitted by the school influence the lives of adolescents and young people. The school congregates a considerable proportion of this population, facilitating access to it. The objective was to analyse the evolution of the AIDS prevention policy for adolescents and young people developed in schools and the role of Unesco.

Method Documentary analysis and interviews. Interviews with health and education managers, CSOs/NGOs and stakeholders to confirm, qualify and complement information produced by documentary analysis.

Results National policy formulated in 1994: School Project (1995-2004); Health and Prevention in School Project (SPE) (2005-2007); Health in School Program (PSE) (2007-2014). Initially with a focus on prevention, the actions passed to integrate a broad set of objectives and contents of health, educational-preventive and care. Large number of schools work the themes; Low effectiveness of educational action. Availability of condoms in schools is little implemented.In the political environment, there is a resurgence of positions that are contrary to discuss sexual diversity at school with a reflection on the activities, mainly considering the interference of conservative political parties and the lobbying of Catholic and Evangelical churches with the decision-making bodies of politics and the management.

Conclusion Young people remain as one of the key populations to control the epidemic. Future of STI and AIDS prevention in litigation schools: Most managers and stakeholders believe that action should be rethought and taken up in isolation or associated with complementary strategies; Others argue that it must be replaced, because it has failed and because the school has become a barrier to young people. UNESCO had a strategic and essential role to promote the approximation of interests and articulation and cooperation between the Ministries of Health and Education.

\subsection{UNDERSTANDING SEXUAL HEALTH LANGUAGE: COMMUNITY ENGAGEMENT WITH REFUGEES FROM BURMA, POST SETTLEMENT IN AUSTRALIA}

${ }^{1}$ Amita Tuteja, ${ }^{2}$ Lena Sanci, ${ }^{3}$ Lester Mascarenhas, ${ }^{4}$ Elisha Riggs, ${ }^{3}$ Lynette 0 Dwyer, ${ }^{3}$ Di Van Villet, ${ }^{3}$ Katrina Sangster, ${ }^{3}$ Kim Mcguiness, ${ }^{2}$ Meredith Temple-Smith. ${ }^{1}$ Department of General Practice, University of Melbourne, Melbourne, Australia; ${ }^{2}$ Department of General Practice, University of Melbourne, Melbourne, Australia; ${ }^{3}$ Isis Primary Care, Hoppers Crossing, Melbourne, Australia; ${ }^{4}$ Murdoch's Children Research Institute, Royal Children's Hospital, University of Melbourne, Melbourne, Australia

\subsection{6/sextrans-2017-053264.13}

Introduction Heath care professionals (HCP) who use interpreters in reproductive health consultations with people of dissimilar cultural backgrounds often describe situations where interpreters and patients who speak the same language fail to convey intended meanings. Interpreters struggle to explain certain words, attempt to substitute taboo words or explain terms with paragraphs. This study aims to engage with refugees from Burma to critically examine the language and vocabulary of sexual health consults.

Methods Using qualitative techniques, we interviewed $27 \mathrm{HCP}$ (doctors, nurses, interpreters, social workers) involved with refugees from Burma. The interview guide drew from a conceptual framework based on principles of "humanization" of reproductive health care interlinked with ideas of "sustainable development". Interviews were audio-recorded and transcribed. 